\title{
Bovine Lentivirus Group
}

National Cancer Institute

\section{Source}

National Cancer Institute. Bovine Lentivirus Group. NCI Thesaurus. Code C117712.

A non-taxonomic grouping of lentivuruses that infect cattle. 